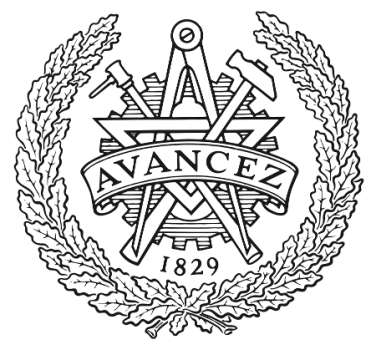

CHALMERS

UNIVERSITY OF TECHNOLOGY

\title{
High-confinement high-Q silicon-rich silicon nitride nonlinear microresonators
}

Downloaded from: https://research.chalmers.se, 2023-04-26 07:50 UTC

Citation for the original published paper (version of record):

Torres Company, V., Ye, Z., Fülöp, A. et al (2018). High-confinement high-Q silicon-rich silicon nitride nonlinear microresonators. Optics InfoBase Conference Papers, Part F101-IPRSN 2018(2018). http://dx.doi.org/10.1364/IPRSN.2018.ITh2J.2

N.B. When citing this work, cite the original published paper. 


\title{
High-confinement high-Q silicon-rich silicon nitride nonlinear microresonators
}

\author{
Victor Torres-Company, Zhichao Ye, Attila Fülöp, Clemens J. Krückel and Peter A. Andrekson \\ Department of Microtechnology and Nanoscience, SE-41296 Gothenburg, Sweden \\ torresv@chalmers.se
}

\begin{abstract}
We show our results on high-Q high-confinement microresonators based on the siliconrich silicon nitride (SiRN) platform, featuring broad anomalous dispersion and an enhanced nonlinear Kerr coefficient with respect to stoichiometric $\mathrm{Si}_{3} \mathrm{~N}_{4}$ technology.

OCIS codes: (130.4310) Nonlinear; (130.2755) Glass waveguides; (190.4390) Integrated optics
\end{abstract}

\section{Introduction}

Silicon nitride $(\mathrm{SiN})$ is a well-established dielectric material in the semiconductor industry that can be easily deposited on silicon wafers to fabricate photonic chips for applications ranging from telecom to biophotonics [1]. This photonic platform has become increasingly relevant for guided-wave nonlinear optics. Its success stems from its ultra-broad transparency window, moderately high linear and nonlinear refractive index coefficients, and the absence of multiphoton absorption in the near infrared [2].

Interestingly, the chemical composition in SiN can be precisely controlled at the deposition process [3]. Although the influence of the film's stoichiometry on the linear refractive index has been widely studied before, its impact on the nonlinear refractive index has only been investigated recently [4-6]. In particular, our group performed a systematic study of the change in refractive index with the low-pressure chemical vapor deposition (LPCVD) parameters. We found an empirical relation between the optical bandgap of the material [7] and the nonlinear refractive index, summarized for convenience in Fig. 1. The bottom line is that a decrease in optical bandgap leads to an increase in both linear and refractive index. The SiN film has a slightly higher content in $\mathrm{Si}$ than stoichiometric SiN, resulting in silicon-rich silicon nitride ( $\mathrm{SiRN}$ ) [8]. In addition, SiRN displays a decrease in the tensile stress of the SiN film, hence the possibility to grow thicker films and attain tighter optical confinement. As a result, the nonlinear Kerr parameter could be enhanced by almost an order of magnitude in silicon-SiRN with respect to stoichiometric SiN [4]. A downside of SiRN that we overcome in this work is that it is usually accompanied by higher linear losses. Here, by a careful optimization of the deposition process and annealing, we achieve high-Q microresonators (intrinsic $\mathrm{Q} \sim 1$ million in the telecom band) in a planar geometry that features broadband anomalous dispersion and high optical confinement. To the best of our knowledge, this is the first time that $\mathrm{SiRN}$ waveguide technology displays an equivalent loss below $1 \mathrm{~dB} / \mathrm{cm}$, thus opening opportunities for nonlinear optics with low-power continuous-wave lasers. We will also present in the conference our recent results [9] illustrating broadband Kerr comb generation in this emerging material platform.

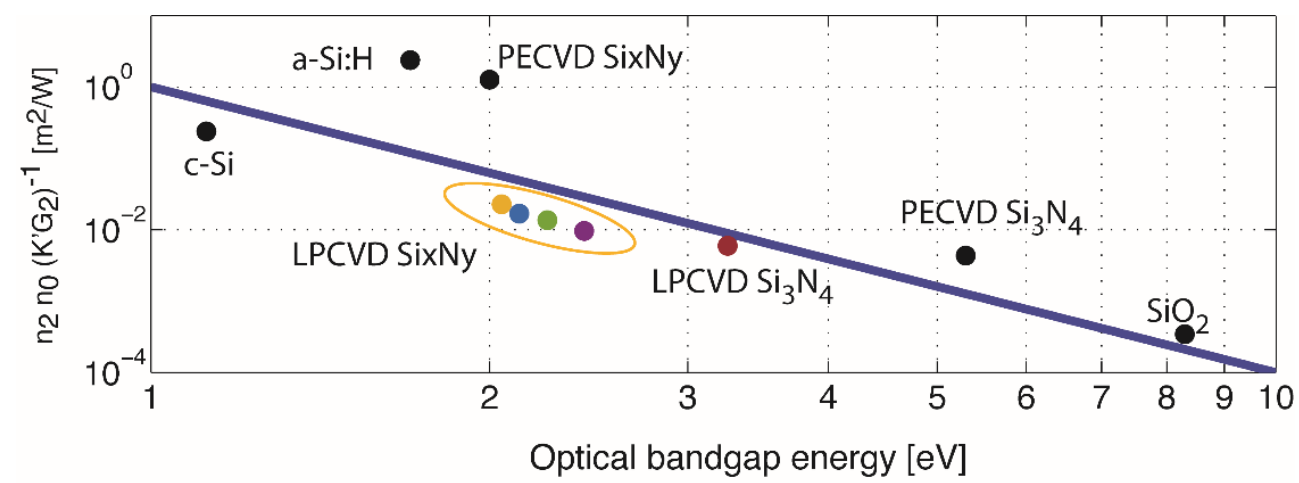

Fig. 1. Comparison of nonlinear Kerr coefficient $\mathrm{n}_{2}$ between silicon-rich silicon nitride and other materials used for nonlinear optics. By controlling the optical bandgap of the material, the nonlinear coefficient can be significantly enhanced with respect to the stoichiometric form. $\mathrm{n}_{0}$ denotes the linear refractive index, $\mathrm{K}^{\prime}$ is a constant and $\mathrm{G}_{2}$ a function that depends on the optical bandgap. Results adapted from [4].

\section{High-Q microresonators based on high-confinement low-loss SiRN waveguide technology}

The fabrication of high-Q microresonators is based on the process reported in [4]. A film of $660 \mathrm{~nm}$ in thickness is deposited via LPCVD at $770{ }^{\circ} \mathrm{C}$. We set the ratio of dichlorosilane to ammonia to $\sim 4$ and found a linear refractive 
index of 2.07 for the film, indicating that the material is only slightly silicon rich. The material performance corresponds to the purple dot in Fig. 1. The pattern for the photonic structures is defined via deep UV lithography. After etching, we applied a standard cleaning process and annealed the devices for 3 hours at $1100{ }^{\circ} \mathrm{C}$ in a nitrogen atmosphere. The devices are cladded with a $3.5 \mu \mathrm{m} \mathrm{SiO}_{2}$ film based on plasma-enhanced chemical vapor deposition. Figure 2(a) shows a scanning electron microscope image of a fabricated microresonator with $20 \mu \mathrm{m}$ radius. Figure 2(b) shows an example of a typical absorption resonance in another resonator. The loaded (intrinsic) Q is $0.8 \times 10^{6}$ $\left(1 \times 10^{6}\right)$ and the mean value of the intrinsic $\mathrm{Q}$ measured across the $\mathrm{C}$ band is $0.9 \times 10^{6}$. These values are still below what can be attained with high-confinement microresonators based on stoichiometric $\mathrm{Si}_{3} \mathrm{~N}_{4}$ [10-12], but the fabrication process involves much less steps. Finally, Fig. 2(c) illustrates the possibility to engineer the dispersion in this material platform by simply changing the waveguide cross-section geometry. Based on this technology, we have recently generated broadband Kerr frequency combs [9].

(a)

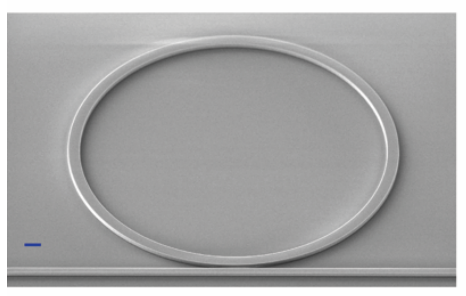

(b)

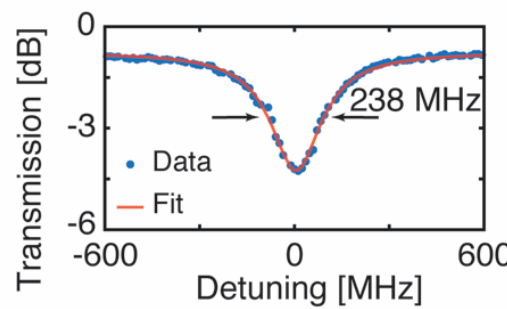

(c)

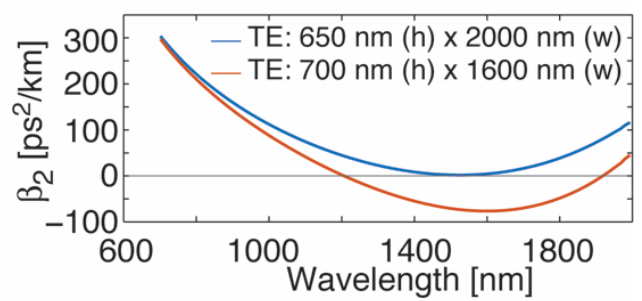

Fig. 2. (a) SEM image of a SiRN microresonator at Chalmers featuring high optical confinement. Scale bar denotes 2 micrometers. (b) typical resonance absorption linewidth. (c) Wavelength dependence of the group velocity dispersion coefficient in the low-loss silicon-rich silicon nitride platform, indicating that normal or anomalous dispersion can be attained over a broad bandwidth by modifying the cross-section geometry of the resonator's waveguide.

\section{Conclusions}

We have demonstrated low-loss high-confinement optical microresonators based on silicon-rich silicon nitride. This platform holds potential for nonlinear optics because of its enhanced nonlinear parameter and decreased film stress, which allows to fabricate thick enough waveguides for high optical confinement. We have reported intrinsic Qs in the order of $\sim 1$ million, indicating an equivalent propagation loss below $1 \mathrm{~dB} / \mathrm{cm}$ in the telecommunications $\mathrm{C}$ band. We will present at the conference our more recent results on Kerr comb generation in this material platform.

\section{References}

[1] A. Rahim et al., "Expanding the silicon photonics portfolio with silicon nitride photonic ....," J. Lightwave Technol. 35, 639-649 (2017).

[2] D. J. Moss et al., "New CMOS-compatible platforms based on silicon nitride and Hydex for nonlinear ...," Nature Photon. 7, 597-607 (2013).

[3] K. N. Andersen et al., “Annealing and deposition effects of the chemical composition of silicon-rich,” Appl. Surf. Sci. 243, 401-408 (2005).

[4] C. J. Krückel et al., “Optical bandgap engineering in nonlinear silicon nitride waveguides,” Opt. Express 25, 15370-15380 (2017).

[5] C. Lacava et al., "Si-rich silicon nitride for nonlinear signal processing applications," Sci. Rep. 7:22 (2017).

[6] K. Koskinen et al., "Enhancement of bulk second-harmonic generation from silicon nitride films by...," Opt. Lett. 42, 5030-5033 (2017).

[7] Note that we define the optical bandgap as the energy in the visible range for which the imaginary component of the bulk refractive index becomes non-zero.

[8] C. J. Krückel et al., "Linear and nonlinear characterization of low-stress high-confinement ...," Opt. Express 23, 25827-25837 (2015).

[9] Z. Ye et al., "Low loss silicon-rich silicon nitride for nonlinear optics" (to be presented at CLEO 2018).

[10] M. H. P. Pfeiffer et al., "Photonic Damascene process for integrated high-Q microresonator based nonlinear...," Optica 3, 20-25 (2016).

[11] Y. Xuan et al., "High-Q silicon nitride microresonators exhibiting low-power frequency comb initiation," Optica 3, 1171-1180 (2016).

[12] X. Ji et al., "Ultra-low-loss on-chip resonators with sub-milliwatt parametric oscillation threshold," Optica 4, 619-624 (2017).

This work has been supported by the Swedish Research Council (VR). 